\title{
A MULTIPLIER RULE IN ABSTRACT SPACES*
}

\author{
HERMAN H. GOLDSTINE
}

The Lagrange multiplier rule has been generalized to certain noncalculus of variations problems by Graves, $\dagger$ Hahn, $\ddagger$ and the author. $\S$ Moreover a very general problem was formulated by L. A. Lusternik. $\|$ However his work seems to rest upon a theorem which is stated without proof and which the author is unable to verify. There are also certain other difficulties with his proof. The problem herein presented is so formulated that the problem of Bolza in the calculus of variations, the problems treated by Graves, Hahn, and the author, and the problem of minimizing a functional defined upon an arbitrary Banach space subject to very general numerically-valued side conditions, and numerous other examples are included as special cases.

The proof proceeds along lines which are essentially generalizations of the methods of the calculus of variations. This demonstration is made possible by the very powerful implicit function theorems of Hildebrandt and Graves $\Psi$ and yields analogs of the transversality condition and of the Euler-Lagrange equations. Some instances which explain the number of linear spaces involved are given in the concluding section.

1. Definitions and assumptions. In order to obtain our statement and proof of the multiplier rule it is convenient to refer to five normed linear spaces $\mathfrak{M}, \mathfrak{N}, \mathfrak{X}, \mathfrak{U}, \mathfrak{B}$, of which $\mathfrak{M}, \mathfrak{U}$, and $\mathfrak{B}$ are assumed to be complete. It is further supposed that $\mathfrak{M}_{0}, \mathfrak{N}_{0}$, and $\mathfrak{X}_{0}$ are regions of their respective spaces. We shall then be concerned with the following basis:

* Presented to the Society, September 10, 1937.

$\dagger A$ transformation of the problem of Lagrange in the calculus of variations, Transactions of this Society, vol. 35 (1933), pp. 675-682.

$\ddagger$ Ueber die Lagrange’sche Multiplikatorenmethode, Sitzungsberichte der Akademie Wien, vol. 131 (1922), pp. 531-550.

$\S$ The minima of functionals with associated side conditions, Duke Mathematical Journal, vol. 3 (1937), pp. 418-425.

|| Sur les extrêmes relatifs des fonctionnelles (in Russian), Recueil Mathématique de la Société Mathématique de Moscou, vol. 41 (1934), pp. 390-401.

I See L. M. Graves, Topics in the functional calculus, this Bulletin, vol. 31 (1935), pp. 641-662; Riemann integration and Taylor's theorem in general analysis; Implicit functions and differential equations in general analysis; and T. H. Hildebrandt and L. M. Graves, Implicit functions and their differentials in general analysis. The last three articles cited appear in the Transactions of this Society, vol. 29 (1927), pp. 163$177,514-552$, and $127-153$, respectively. In the sequel these papers will be referred to as papers I to IV, respectively. 
A. $f$ and $\psi_{k},(k=1, \cdots, q)$, where $q$ is an arbitrary integer, are real-valued functions defined on the composite* $\left(\mathfrak{X}_{0}, \mathfrak{M}_{0}\right)$ of $\mathfrak{X}_{0}$ and $\mathfrak{M}_{0}$, each of which has a first differential at some fixed point $\left(\bar{x}_{0}, \bar{\mu}_{0}\right)$. Moreover the functionals $\psi_{k},(k=1, \cdots, q)$, vanish at this point $\left(\bar{x}_{0}, \bar{\mu}_{0}\right)$.

B. $u=\phi_{1}\left(x_{0}, \mu_{0}, \nu_{0}\right)$ is an operation of class $C^{\prime}$ defined on $\left(\mathfrak{X}_{0}, \mathfrak{M}_{0}, \mathfrak{N}_{0}\right)$, and having its functional values in $\mathfrak{U}$; and $\nu_{0}=n\left(x_{0}, \mu_{0}\right)$ is defined over $\left(\mathfrak{X}_{0}, \mathfrak{M}_{0}\right)$, has its values in $\mathfrak{N}_{0}$, is of class $C^{\prime}$, and is such that $\phi_{1}\left[\bar{x}_{0}, \bar{\mu}_{0}, n\left(\bar{x}_{0}, \bar{\mu}_{0}\right)\right]=0_{u}$, the zero element in the space $\mathfrak{U}$. (Analogous notations will be used for the zero points in the various spaces treated.)

C. There is an operation $v=\phi_{2}\left(x_{0}, \mu_{0}, \nu_{0}\right)$ of class $C^{\prime}$, which is defined on a neighborhood of the point $\left(\bar{x}_{0}, \bar{\mu}_{0}, \bar{\nu}_{0}\right)$, where $\bar{\nu}_{0}=n\left(\bar{x}_{0}, \bar{\mu}_{0}\right)$, and has its functional values in $\mathfrak{B}$, such that the linear and continuous function of $\mu,\left(d_{\mu} \phi_{1}\left(x_{0}, \mu_{0}, \nu_{0} ; \mu\right), d_{\mu} \phi_{2}\left(x_{0}, \mu_{0}, \nu_{0} ; \mu\right)\right)$, has a linear continuous reciprocal at $\left(x_{0}, \mu_{0}, \nu_{0}\right)=\left(\bar{x}_{0}, \bar{\mu}_{0}, \bar{\nu}_{0}\right)$.

D. The functional $f$ has a minimum at $\left(\bar{x}_{0}, \bar{\mu}_{0}\right)$ in the class of points in $\left(\mathfrak{X}_{0}, \mathfrak{M}_{0}\right)$ which satisfy the equations

$$
\begin{aligned}
\phi_{1}\left[x_{0}, \mu_{0}, n\left(x_{0}, \mu_{0}\right)\right] & =0_{u}, \\
\psi_{k}\left(x_{0}, \mu_{0}\right) & =0, \quad k=1, \cdots, q .
\end{aligned}
$$

In order to simplify the notation the element $\bar{v}$ of $\mathfrak{B}$ will be used to designate $\phi_{2}\left(\bar{x}_{0}, \bar{\mu}_{0}, \bar{\nu}_{0}\right) ; \Phi_{\alpha}(x, \mu, \nu)$ to indicate $d \phi_{\alpha}\left(\bar{x}_{0}, \bar{\mu}_{0}, \bar{\nu}_{0} ; x, \mu, \nu\right)$, $(\alpha=1,2)$; and $N(x, \mu)$ to connote $d n\left(\bar{x}_{0}, \bar{\mu}_{0} ; x, \mu\right)$.

2. The imbedding theorem. It is of paramount importance for our purposes to show that the minimizing element $\left(\bar{x}_{0}, \bar{\mu}_{0}\right)$ is a limit point of elements $\left(x_{0}, \mu_{0}\right)$ which satisfy the equation

$$
\phi_{1}\left[x_{0}, \mu_{0}, n\left(x_{0}, \mu_{0}\right)\right]=0_{u} .
$$

To show that the element $\left(\bar{x}_{0}, \bar{\mu}_{0}\right)$ is so imbedded it is convenient first to demonstrate several lemmas.

LEMмa 2.1. In a sufficiently small neighborhood of $\bar{\mu}_{0}$ the equations

$$
\begin{aligned}
& \phi_{1}\left(x_{0}, \mu_{0}, \nu_{0}\right)=u, \\
& \phi_{2}\left(x_{0}, \mu_{0}, \nu_{0}\right)=v
\end{aligned}
$$

have, for each $\left(x_{0}, \nu_{0}, u, v\right)$ in a neighborhood of $\left(\bar{x}_{0}, \bar{\nu}_{0}, 0_{u}, \bar{v}\right)$, a unique solution, $\mu_{0}=M_{1}\left(x_{0}, \nu_{0}, u, v\right)$, of class $C^{\prime}$.

This result follows at once from the implicit function theorems

* See paper IV for the definitions of terms such as composite, neighborhood, region, differential. 
mentioned above, ${ }^{*}$ because of assumptions $C$ and the fact that $\left(\mu_{0}, x_{0}, \nu_{0}, u, v\right)=\left(\bar{\mu}_{0}, \bar{x}_{0}, \bar{\nu}_{0}, 0_{u}, \bar{v}\right)$ is an initial solution of these equations.

Corollary 2.2. For each $(x, \nu, U, V)$ the equations

$$
\begin{aligned}
& \Phi_{1}(x, \mu, \nu)=U, \\
& \Phi_{2}(x, \mu, \nu)=V
\end{aligned}
$$

have a unique continuous solution $\mu=M_{2}(x, \nu, U, V)$ which is equal to $d M_{1}\left(\bar{x}_{0}, \bar{\nu}_{0}, 0_{u}, \bar{v} ; x, \nu, U, V\right)$.

The existence and unicity of the solution is an immediate consequence of assumption $C$; and the last assertion in the corollary can be established by setting $x_{0}=\bar{x}_{0}+a x, \nu_{0}=\bar{\nu}_{0}+a \nu, u=a U, v=\bar{v}+a V$, and $\mu_{0}=M_{1}\left(x_{0}, \nu_{0}, u, v\right)$ in equations (2.1) and differentiating the resulting identities in $a$ at $a=0$.

Then to obtain the desired imbedding theorems we make a final assumption.

E. Either the function $M_{1}$, mentioned above, is independent of $\nu_{0}$, or the transformation

$$
\mu-d_{\nu} M_{1}\left[\bar{x}_{0}, \bar{\nu}_{0}, 0_{u}, \bar{v} ; d_{\mu} n\left(\bar{x}_{0}, \bar{\mu}_{0} ; \mu\right)\right]
$$

has a linear continuous reciprocal.

It follows readily from Lemma 2.1 that if $M_{1}$ is independent of $\nu_{0}$ and if $M\left(x_{0}, u, v\right)$ is defined to be $M_{1}\left(x_{0}, \nu_{0}, u, v\right)$, then the functions $\phi_{1}\left[x_{0}, M\left(x_{0}, u, v\right), \nu_{0}\right], \phi_{2}\left[x_{0}, M\left(x_{0}, u, v\right), \nu_{0}\right]$ are independent of $\nu_{0}$, at least for $\left(x_{0}, \nu_{0}, u, v\right)$ sufficiently near to $\left(\bar{x}_{0}, \bar{\nu}_{0}, 0_{u}, \bar{v}\right)$.

Theorem 1. In a sufficiently small neighborhood of $\vec{\mu}_{0}$ the equations

$$
\begin{aligned}
& \phi_{1}\left[x_{0}, \mu_{0}, n\left(x_{0}, \mu_{0}\right)\right]=u, \\
& \phi_{2}\left[x_{0}, \mu_{0}, n\left(x_{0}, \mu_{0}\right)\right]=v
\end{aligned}
$$

possess a unique solution $\mu_{0}=M\left(x_{0}, u, v\right)$ of class $C^{\prime}$ for $\left(x_{0}, u, v\right)$ in some neighborhood of $\left(\bar{x}_{0}, 0_{u}, \bar{v}\right)$.

From what has been said above it suffices to consider the case where $M_{1}$ is not independent of $\nu_{0}$. Hence the equation

$$
\mu_{0}=M_{1}\left[x_{0}, n\left(x_{0}, \mu_{0}\right), u, v\right]
$$

has a solution $\mu_{0}=M\left(x_{0}, u, v\right)$ which is effective in the theorem.

Similarly a consideration of the equation

$$
\mu=d M_{1}\left[\bar{x}_{0}, \bar{\nu}_{0}, 0_{u}, \bar{v} ; x, N(x, \mu), U, V\right]
$$

\footnotetext{
* See paper I, p. 65, and paper IV, p. 150.
} 
yields the following corollary:

Corollary 2.3. For each $(x, U, V)$ the equations

$$
\begin{aligned}
& \Phi_{1}[x, \mu, N(x, \mu)]=U, \\
& \Phi_{2}[x, \mu, N(x, \mu)]=V
\end{aligned}
$$

have a unique continuous solution $\mu=M_{3}(x, U, V)=d M\left(\bar{x}_{0}, 0_{u}, \bar{v}\right.$; $x, U, V)$.

CoRollary 2.4. Let $s$ be an arbitrary integer, $\left(x_{1}, \cdots, x_{s}\right)$ a set of elements of $\mathfrak{X}$, and $\left(\mu_{1}, \cdots, \mu_{s}\right)$ a set of elements of $\mathfrak{M}$ salisfying $\Phi_{1}[x, \mu, N(x, \mu)]=0_{u}$. Then there are functions $x_{0}(b)$ and $\mu_{0}(b)$ of class $C^{\prime}$ for values of $b=\left(b_{1}, \cdots, b_{s}\right)$ sufficiently near to $(0, \cdots, 0)$, which satisfy the equation

$$
\phi_{1}\left[x_{0}, \mu_{0}, n\left(x_{0}, \mu_{0}\right)\right]=0_{u},
$$

$x_{0}(0)=x_{0}, \mu_{0}(0)=\bar{\mu}_{0}$, and $\partial x_{0}(0) / \partial b_{i}=x_{i}, \partial \mu_{0}(0) / \partial b_{i}=\mu_{i}$ for $1 \leqq i \leqq s$.

Define $V_{i}$ to be $\Phi_{2}\left[x_{i}, \mu_{i}, N\left(x_{i}, \mu_{i}\right)\right],(i=1, \cdots, s), x_{0}(b)$ $=x_{0}\left(b_{1}, \cdots, b_{s}\right)=\bar{x}_{0}+b_{i} x_{i}$, and $\mu_{0}(b)$ to be $M\left[x_{0}(b), 0_{u}, \bar{v}+b_{i} V_{i}\right]$, where a repeated index indicates a sum. Then the first part of the corollary is obvious, and the latter part is an easy consequence of corollary 2.3 .

3. The multiplier rule. The usual mode of proof utilized in the calculus of variations suffices to establish the following lemma:

Lemma 3.1. There are constants $l_{0}, c_{1}, \cdots, c_{q}$, not all zero, such that

$$
g(x, \mu)=l_{0} d f\left(\bar{x}_{0}, \bar{\mu}_{0} ; x, \mu\right)+c_{k} d \psi_{k}\left(\bar{x}_{0}, \bar{\mu}_{0} ; x, \mu\right)=0
$$

for every $(x, \mu)$ satisfying the equation

$$
\Phi_{1}[x, \mu, N(x, \mu)]=0_{u} .
$$

To simplify the succeeding demonstration we shall define $g_{1}(x)$ and $g_{2}(\mu)$ to be $l_{0} d_{x} f\left(\bar{x}_{0}, \bar{\mu}_{0} ; x\right)+c_{k} d-\psi_{k}\left(\bar{x}_{0}, \bar{\mu}_{0} ; x\right)$ and $l_{0} d_{\mu} f\left(\bar{x}_{0}, \bar{\mu}_{0} ; \mu\right)$ $+c_{k} d_{\mu} \psi_{k}\left(\bar{x}_{0}, \bar{\mu}_{0} ; \mu\right)$, respectively. It is then obvious that $g(x, \mu)=g_{1}(x)$ $+g_{2}(\mu)$ and that $g_{1}$ and $g_{2}$ are linear continuous functionals on $\mathfrak{X}$ and $\mathfrak{M}$, respectively.

Lemma 3.2. The functions $\Phi_{1}\left[x, M_{3}(x, U, V)\right], N\left[x, M_{3}(x, U, V)\right]$, and $g\left[x, M_{3}(x, U, V)\right]$ are independent of $x$ and $V$; the latter is a linear continuous functional $J$ on $\mathfrak{U}$; and for every $x$

$$
g_{1}(x)+g_{2}\left[M_{3}\left(x, 0_{u}, 0_{v}\right)\right]=0 .
$$


It follows at once from Corollary 2.3 that $\Phi_{1}$ is independent of $x$ and $V$. Hence we have $\Phi_{1}[\bar{x}, \bar{\mu}, N(\bar{x}, \bar{\mu})]=0_{u}$, where $\bar{x}=x_{1}-x_{2}$, and $\bar{\mu}=M_{3}\left(\bar{x}, 0_{u}, V_{1}-V_{2}\right)$. Consequently we have, by Lemma $3.1, g(\bar{x}, \bar{\mu})$ $=0$ for every $x_{1}, x_{2}, V_{1}$, and $V_{2}$; this proves the rest of the lemma, since $M_{3}\left(x_{1}, U, V_{1}\right)-M_{3}\left(x_{2}, U, V_{2}\right)=M_{3}\left(x_{1}-x_{2}, 0_{u}, V_{1}-V_{2}\right)$.

To complete the proof of the multiplier rule we shall now prove that there is a linear continuous operation $u=P(\mu)$ on $\mathfrak{M}$ to $\mathfrak{U}$ such that $g_{2}(\mu)=J[P(\mu)]$ for every $\mu$. To this end define $P(\mu)$ to be $\Phi_{1}\left[0_{x}, \mu, N\left(0_{x}, \mu\right)\right]$ and $Q(\mu)$ to be $\Phi_{2}\left[0_{x}, \mu, N\left(0_{x}, \mu\right)\right]$. Therefore, by Corollary 2.3, $\mu=M_{3}\left[0_{x}, P(\mu), Q(\mu)\right]$; as a consequence it is evident that $g_{2}(\mu)=g_{2}\left(M_{3}\left[0_{x}, P(\mu), Q(\mu)\right]\right)$ which, by Lemma 3.2, is $J[P(\mu)]$.

THEOREM 2. There exist constants $l_{0}, c_{1}, \cdots, c_{q}$, not all zero, and a linear, continuous, and real-valued functional $J$ on $\mathfrak{U}$ such that

$$
l_{0} d_{\mu} f\left(\bar{x}_{0}, \bar{\mu}_{0} ; \mu\right)+c_{k} d_{\mu} \psi_{k}\left(\bar{x}_{0}, \bar{\mu}_{0} ; \mu\right)-J\left(\Phi_{1}\left[0_{x}, \mu, N\left(0_{x}, \mu\right)\right]\right)=0
$$

for every $\mu$ in $\mathfrak{M}$, and

$$
l_{0} d_{x} f\left(\bar{x}_{0}, \bar{\mu}_{0} ; x\right)+c_{k} d_{x} \psi_{k}\left(\bar{x}_{0}, \bar{\mu}_{0} ; x\right)-J\left(\Phi_{1}\left[x, 0_{\mu}, N\left(x, 0_{\mu}\right)\right]\right)=0
$$

for every $x$ in $\mathfrak{X}$.

From the previous discussion it is evident that

$$
g_{2}(\mu)=l_{0} d_{\mu} f\left(\bar{x}_{0}, \bar{\mu}_{0} ; \mu\right)+c_{k} d_{\mu} \psi_{k}\left(\bar{x}_{0}, \bar{\mu}_{0} ; \mu\right)=J[P(\mu)]
$$

and that $P(\mu)$ is $\Phi_{1}\left[0_{x}, \mu, N\left(0_{x}, \mu\right)\right]$. Equation (2), the "transversality condition," follows from equation (3.1). For,

$$
g_{2}\left[M_{3}\left(x, 0_{u}, 0_{v}\right)\right]=J\left(P\left[M_{3}\left(x, 0_{u}, 0_{v}\right)\right]\right) .
$$

Then making use, on the one hand, of the definitions of $P$ and $\Phi_{1}$, and on the other hand of Corollary 2.3, we obtain the desired conclusion.

4. Instances of the theory. We shall illustrate the generality of our theory by working out two special cases, the minima of functions of several variables and the problem of Bolza in the calculus of variations. However the theory also includes the situations treated by Hahn, Graves, and the author, as well as many others.

Consider first the problem of minimizing a real-valued function $g\left(x_{1}, \cdots, x_{m}\right)$ of $m$ real variables in the class of points $x_{0}=\left(x_{1}, \cdots, x_{m}\right)$ satisfying equations $h_{k}\left(x_{0}\right)=0,(k=1, \cdots, q)$. It is assumed that $g$ has a minimum at a point $\bar{x}_{0}$ in this class and that $g$ and $h_{k}$ have first differentials at this point. Then to indicate the applicability of our theory to this case we choose the sets $\mathfrak{M}, \mathfrak{N}, \mathfrak{M}_{0}, \mathfrak{N}_{0}, \mathfrak{U}$, and $\mathfrak{B}$ to be equal to the set of real numbers, $\mathfrak{X}$ to be the $m$-dimensional euclidean space, and $\mathfrak{X}_{0}$ to be the region of definition of 
the functions $g$ and $h_{1}, \cdots, h_{q}$. We then make the following definitions: $\psi_{k}\left(x_{0}, \mu_{0}\right) \equiv h_{k}\left(x_{0}\right),(k=1, \cdots, q), f\left(x_{0}, \mu_{0}\right) \equiv g\left(x_{0}\right), n\left(x_{0}, \mu_{0}\right) \equiv 0$, $\phi_{1}\left(x_{0}, \mu_{0}, \nu_{0}\right) \equiv \mu_{0}$, and $\bar{\mu}_{0} \equiv 0$. Then it is clear that $f\left(x_{0}, \mu_{0}\right)$ is a minimum at $\left(\bar{x}_{0}, \bar{\mu}_{0}\right)$ in the class of all points $\left(x_{0}, \mu_{0}\right)$ in the region which satisfy

$$
\begin{aligned}
\psi_{k}\left(x_{0}, \mu_{0}\right) & =0, \quad k=1, \cdots, q, \\
\mu_{0} & =0,
\end{aligned}
$$

and all previous assumptions are satisfied.

Our multiplier rule then implies the existence of constants $l_{0}, c_{1}, \cdots, c_{q}$, not all zero, and a constant $e$ such that

$$
l_{0} g_{x_{i}}\left(\bar{x}_{0}\right) d x_{i}+c_{k} h_{k x_{i}}\left(\bar{x}_{0}\right) d x_{i}=0, \quad e d \mu=0
$$

for every $d x_{1}, \cdots, d x_{m}$ and every $d \mu$; therefore we have $e=0$ and

$$
l_{0} g_{x_{i}}\left(\bar{x}_{0}\right)+c_{k} h_{x_{i}}\left(\bar{x}_{0}\right)=0, \quad i=1, \cdots, m,
$$

which is the familiar form of the multiplier rule for this problem. The problem of minimizing a function $f$ on a region $\mathfrak{X}_{0}$ of a normed linear space in the class of points satisfying functional equations $\psi_{k}\left(x_{0}\right)=0,(k=1, \cdots, q)$, can be treated in precisely the manner indicated above.

Let us turn our attention to the problem of Bolza in the calculus of variations, where, for simplicity, we shall suppose that the admissible arcs are of class $C^{\prime}$. Here we are given an arc $\bar{y}_{1}(x), \cdots, \bar{y}_{s}(x)$, $\left(\bar{x}_{1} \leqq x \leqq \bar{x}_{2}\right)$, which minimizes a functional

$$
I\left[x_{1}, x_{2}, y, y^{\prime}\right]=g\left[x_{1}, y\left(x_{1}\right), x_{2}, y\left(x_{2}\right)\right]+\int_{x_{1}}^{x_{2}} h\left[x, y(x), y^{\prime}(x)\right] d x
$$

in the class of admissible arcs which satisfy certain side conditions

$$
\phi_{\alpha}\left[x, y(x), y^{\prime}(x)\right]=0, \quad \alpha=1, \cdots, r<s,
$$

and end conditions

$$
\psi_{k}\left[x_{1}, y\left(x_{1}\right), x_{2}, y\left(x_{2}\right)\right]=0, \quad k=1, \cdots, q .
$$

For our purposes it is sufficient to restrict attention to the class $\mathfrak{N}_{0}$ of all admissible sets of functions $\left(y_{1}(x), \cdots, y_{s}(x)\right)$, $\left(\bar{x}_{1}-e \leqq x \leqq \bar{x}_{2}+e\right)$, where $e$ is some fixed positive constant. The class which is composed of all sets $\left(y_{1}^{\prime}(x), \cdots, y_{s}^{\prime}(x)\right)$ of derivatives of an element $\nu_{0}=\left(y_{1}(x), \cdots, y_{s}(x)\right)$ is $\mathfrak{M}_{0}$. Then if the transformation

$$
y_{i}(x)=y_{i}\left(\bar{x}_{1}\right)+\int_{\bar{x}_{1}}^{x} y_{i}^{\prime}(t) d t
$$


is performed upon the functionals $I$ and $\psi_{k}$, they become functionals $f\left(x_{0}, \mu_{0}\right)$ and $\psi_{k}\left(x_{0}, \mu_{0}\right)$ of $x_{0}=\left(x_{1}, x_{2}, y\left(\bar{x}_{1}\right)\right), \mu_{0}=\left(y^{\prime}\right)$. Thus $\mathfrak{X}_{0}$ is the set of all $\left(x_{1}, x_{2}, y_{01}, \cdots, y_{0 s}\right)$, where $\left|\bar{x}_{1}-x_{1}\right|<e,\left|\bar{x}_{2}-x_{2}\right|<e$, and $\left(y_{01}, \cdots, y_{0_{8}}\right)$ is the value of an admissible set at $x=\bar{x}_{1}$.

To complete our transformation of the problem of Bolza we define $\phi_{1}\left(x_{0}, \mu_{0}, \nu_{0}\right)$ to be the set of $m$ functions

$$
\phi_{1}\left[x, \nu_{0}(x), \mu_{0}(x)\right], \cdots, \phi_{m}\left[x, \nu_{0}(x), \mu_{0}(x)\right]
$$

and $n\left(x_{0}, \mu_{0}\right)$ to be the set of $s$ functions of $\left(x_{0}, \mu_{0}\right)$ which appear in the right members of equations (4.1). It is then a simple matter to verify assumptions A through $D$ of $\$ 1$, if it is assumed that the matrix $\phi_{\alpha y_{i}}$ of partial derivatives has rank $m$ along the minimizing arc. Assumption $\mathrm{E}$ can be verified without difficulty by making use of the theory of Volterra integral equations.

The functional $J$ appearing in our multiplier rule can be shown* to be representable as

$$
-\int_{\bar{x}_{1}}^{\bar{x}_{2}} u_{\alpha}(x) \lambda_{\alpha}(x) d x .
$$

Now, if we define $F$ to be $l_{0} h+\lambda_{\alpha} \phi_{\alpha}$, identity (1) of Theorem 2 yields the following equations:

$$
\begin{aligned}
F_{y_{i}}(x) d x+l_{0}\left(g_{y_{i 1}}+g_{y_{i 2}}\right)+c_{k}\left(\psi_{k y_{i 1}}+\psi_{k y_{i 2}}\right) & =0, \\
(-1)^{b} F\left(\tilde{x}_{b}\right)+l_{0}\left[g_{x_{b}}+g_{y_{i b}} y_{i}^{\prime}\left(\bar{x}_{b}\right)\right]+c_{k}\left[\psi_{k x_{b}}+\psi_{k y_{i b}} y_{i}^{\prime}\left(\bar{x}_{b}\right)\right] & =0, \\
b & =1,2 ;
\end{aligned}
$$

moreover identity (2) becomes

$$
F_{y_{i^{\prime}}}(x)-\int_{\bar{z}_{2}}^{x} F_{y_{i}}(t) d t+\log _{y_{i_{2}}}+c_{k} \psi_{k y_{y_{2}}}=0 .
$$

By very obvious manipulations these equations can be put into the more familiar form

$$
\begin{gathered}
{\left[\left(F-y_{i}^{\prime} F_{\left.y_{i^{\prime}}\right) d x}+F_{y_{i}{ }^{\prime}} d y_{i}\right]_{1}^{2}+l_{0} d g+c_{k} d \psi_{k}=0,\right.} \\
F_{y_{i^{\prime}}}=\int_{\bar{x}_{1}}^{x} F_{y_{i}} d x+d_{i},
\end{gathered}
$$

which is the multiplier rule for the problem of Bolza.

The University of Chicago

\footnotetext{
* See Hahn, loc. cit., pp. 544-546.
} 